\title{
Infective endocarditis caused by Arcanobacterium haemolyticum: a case report
}

Vanessa Wong ${ }^{1 *}$, Tom Turmezei ${ }^{2}$, Maria Cartmill ${ }^{3}$ and Shiu Soo ${ }^{1}$

\begin{abstract}
Arcanobacterium haemolyticum is an organism that commonly causes pharyngitis and wound infections. It does not usually cause systemic invasive disease. The organism presents a difficult diagnostic problem because the Clinical Microbiology laboratory has a propensity to view them as diphtheroid organisms of the Corynebacterium species, thus contaminants or normal flora. We describe a case of a 21-year-old female who had endocarditis with cerebral emboli due to Arcanobacterium haemolyticum. This rare condition is associated with significant mortality and to the best of our knowledge; this is the first successfully treated case of A. haemolyticum endocarditis complicated by embolic phenomenon.
\end{abstract}

\section{Introduction}

Arcanobacterium haemolyticum is a facultative anaerobic gram-positive bacillus. Originally classified in the Cornyebacterium genus, it was re-classified in a new genus in 1982 [1]. It has been isolated from the skin and pharynx of healthy individuals and it is a wellrecognised cause of pharyngitis, skin and soft-tissue infections [2]. Less commonly, the organism causes deep-seated infections including osteomyelitis [3], brain abscesses [4] and endocarditis [5-7].

Arcanobacterium means 'mysterious bacterium', which is an epithet quite befitting for an organism that is frequently overlooked by the Clinical Microbiology laboratory because it is deemed to be a contaminant or normal flora [8]. Recognition of the ability of this organism to cause disease is important in order to make a correct diagnosis and commence appropriate antibiotic therapy.

Here we describe the first case of successfully treated A. haemolyticum infective endocarditis complicated with cerebral emboli and review the features of this rarely pathogenic organism.

\section{Case report}

A 21-year-old Caucasian female with known congenital heart disease presented with a five-day history of fever, lethargy and a swollen, painful left calf. Her past medical

\footnotetext{
* Correspondence: vanessawong@doctors.org.uk

'Department of Microbiology, Queen's Medical Centre, Derby road, Nottingham, NG7 2UH, UK

Full list of author information is available at the end of the article
}

history included previous surgical repair for pulmonary atresia, quadricuspid aortic valve and a ventricular septal defect (VSD) as a child for which she took life-long warfarin anticoagulation therapy. She had also suffered recurrent miscarriages.

On examination she was febrile at $38.5^{\circ} \mathrm{C}$, but hemodynamically stable. Significant positive findings were a swollen, tender left lower limb and an ejection flow murmur across the prosthetic aortic valve and pulmonary regurgitation through the pulmonary conduit, consistent with her heart condition. The remainder of the clinical examination was unremarkable.

Laboratory results showed: hemoglobin $9.3 \mathrm{~g} / \mathrm{dl}$; mean cell volume $0.32 \mathrm{fl}$; white cell count $8.6 \times 10^{9} /$ liter; neutrophil count $6.7 \times 10^{9} /$ liter; platelet count $268 \times 10^{9} /$ liter; Creactive protein $35 \mathrm{mg} /$ liter; INR 3.0. Her renal and liver function tests were normal. The admission chest radiograph was unremarkable. Ultrasound of her calf showed a hematoma, which was aspirated percutaneously under ultrasound guidance, the subsequent culture being negative.

However, due to ongoing fevers the patient underwent a transthoracic echocardiogram (the patient was initially unable to tolerate the transesophageal approach) that raised the possibility of vegetations on the mitral valve and VSD patch. The patient then suffered a grand mal seizure requiring intubation and admission to the Intensive Care Unit. On examination a fixed and dilated right pupil was noted. Computerized tomography of the head showed a large right frontal parenchymal hematoma 


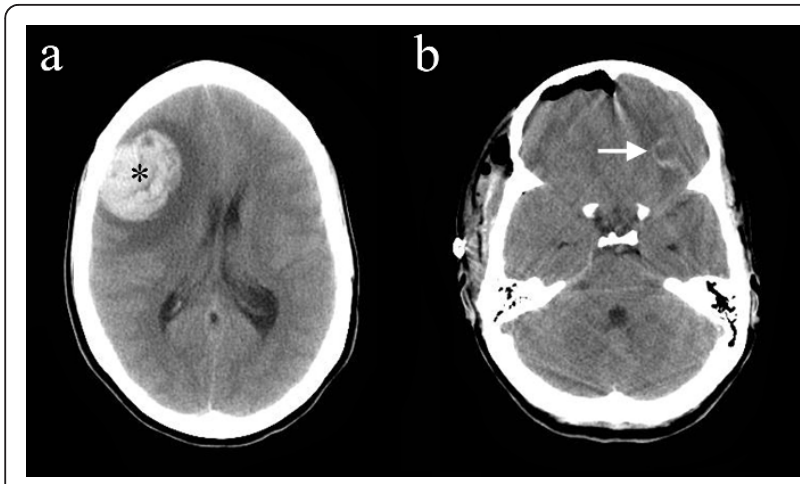

Figure 1 a Axial image from the initial unenhanced CT brain study showing a large heterogeneous high density lesion in the right frontal brain parenchyma consistent with an acute hematoma (asterisk) with the surrounding low density of vasogenic edema causing midline shift to the left. The presence of several smaller ring lesions (see b) combined with the clinical history led the reporting neuroradiologist to raise the possibility of intracranial mycotic aneurysms as well as cerebral abscesses. b Unenhanced axial CT brain image post-craniotomy and hematoma evacuation (note the pneumocephalus). This demonstrates one of the ring lesions in the inferior left frontal region (arrow) that was noted in the initial study. This showed rim enhancement with intravenous contrast administration (not shown), in keeping with the diagnosis of a cerebral abscess.

with several smaller frontal abscesses associated with mass effect and midline shift to the left (Figure 1a).

Neurosurgical review led to an emergency right frontal craniotomy with evacuation of the hematoma (Figure 1b), which was negative on microbiological culture. She was commenced on empirical intravenous ceftriaxone $(2 \mathrm{~g}$ every $12 \mathrm{~h}$ ), vancomycin (1 g every $12 \mathrm{~h}$ ) and metronidazole (500 mg every $8 \mathrm{~h}$ ) on advice from the Microbiology Department. Her post-operative progress was complicated by the need for further neurosurgery due to persistently raised intracranial pressure, resulting in a craniostomy.

Subsequently, 15 days into her admission one out of a total of 12 sets of blood culture specimens became positive after 22 hours of incubation on the BACTEC 9240 system (Becton Dickinson Microbiology Systems Ltd, USA). Pleomorphic gram-positive bacilli were seen in the blood gram film. The blood was inoculated onto several agar plates including: $5 \%$ horse blood agar (HBA; CM0271, Oxoid, UK); fastidious anaerobic agar (FAA; Oxoid, UK); Columbia blood agar with neomycin (FNEO; Oxoid, UK) aerobically and anaerobically for 2 and 5 days respectively. After 48 hours at $37^{\circ} \mathrm{C}$ both aerobic and anaerobic cultures yielded small translucent, non-pigmented colonies, surrounded by a small zone of beta-hemolysis on horse blood agar. The organism was noted to be catalase-negative and was identified as A. haemolyticum (99.9\% probability) by biochemical testing with the API CORYNE identification strips (BioMérieux, France). This was later confirmed by the
Health Protection Agency in London, United Kingdom, using $16 \mathrm{~S}$ rRNA gene sequencing.

Antibiotic susceptibility testing by disk diffusion assay demonstrated that the organism was susceptible to penicillin, ceftriaxone, ciprofloxacin, gentamicin, rifampicin, and vancomycin. The minimal inhibitory concentration (MIC) of penicillin was $0.023 \mathrm{mg} / \mathrm{L}$ to the isolate (penicillin control MIC was $1.5 \mathrm{mg} / \mathrm{L}$ ). There have been several reports of $A$. haemolyticum infective endocarditis in which there have been treatment failures with penicillin [5-7]. In view of this and the fact the patient was clinically improving, Microbiology advised continuing ceftriaxone with the addition of gentamicin $(80 \mathrm{mg}$ every 12 hours) to the treatment regime. Vancomycin and metronidazole were stopped at this point, having now received two weeks therapy of these. A course of 42 days of antibiotics was given in total, with all repeat blood cultures being negative.

On completion of the antibiotic course, the patient had some mild residual weakness and resting tremor of her right lower limb. Repeat transthoracic and transesophageal echocardiograms showed no evidence of endocarditic phenomena. At six months follow-up the patient reported no new symptoms and there was no clinical evidence of relapse of infective endocarditis. Subsequently the patient underwent a successful insertion of intracranial titanium plates and titanium cranioplasty nine months later.

\section{Discussion}

Arcanobacterium haemolyticum was first isolated from pharyngitis and skin infections in American soldiers and native islanders in the South Pacific in 1946 [9]. The organism is often overlooked as part of the normal oral flora, however it has been found in symptomatic individuals either as a sole pathogen or a component of polymicrobial infection $[10,11]$. No risk factors for infection have yet been identified [4,12], although two distinct patient subsets are recognized: healthy young adults presenting with upper respiratory tract infections and older, often immunocompromised, patients presenting with skin and soft tissue infections [11].

Although A. haemolyticum can cause infective endocarditis, to the best of our knowledge only three cases have been reported in the medical literature: the first case was an 87-year-old gentleman who died after developing an infection on a bicuspid aortic valve [7]; the second case was a 50-year-old intravenous drug user (IVDU) who developed mitral valve infective endocarditis and died as a result of cerebral emboli [6] and the third case was a 33-year-old IVDU with HIV-1 infection who survived after developing infective endocarditis of his tricuspid valve without any complication [5]. Our case report describes a young woman with congenital 
heart disease that survived despite developing neurological complications requiring surgery.

After 48 hours incubation colonies display beta-hemolysis that is best observed on 5\% human blood agar [13]. The growth can be optimized by the presence of $5-8 \%$ carbon dioxide, blood or serum-enriched medium and incubation at $37^{\circ} \mathrm{C}$ [14]. There are two distinct biotypes of A. haemolyticum: smooth or rough colonies on solid growth medium. Smooth-type colonies appear even, beta-hemolytic, beta-glucuronidase negative and ferment both sucrose and trehalose. Rough-type colonies appear uneven, non-hemolytic, beta-glucuronidase positive, and do not ferment sucrose or trehalose. The majority of strains are of the smooth-type. Clinically there is a difference between the biotypes with the smooth-type predominately causing wound infections, while the roughtype is isolated almost exclusively from respiratory specimens [15].

There are no established guidelines for the treatment of this infection, although it has been reported to be sensitive to penicillin, cephalexin, erythromycin, and clindamycin, yet resistant to sulfamethoxazole-trimethoprim [16]. Cephalosporins have been found to be reasonable first line agents for deep-seated infections because they are bactericidal. They have good tissue penetration in systemic infections and low rates of resistance have been reported [14]. In addition, the organism has also demonstrated high susceptibility to gentamicin [17]. There have been reports of treatment failure with penicillin, with penicillin tolerance being described on in vitro testing. In infective endocarditis $A$. haemolyticum has also demonstrated both penicillin and ampicillin tolerance, resulting in no clinical improvement on empirical therapy of ampicillin plus gentamicin [5]. Therefore it is prudent to be aware of tolerance to penicillins when deciding on an antibiotic regime with this organism.

\section{Conclusions}

Given the serious morbidity associated with disseminated A. haemolyticum infection and the difficulties that face a Clinical Microbiology laboratory in recognizing it as a human pathogen, it is important to be aware of its potential role in causing bloodstream infections, particularly in patients with predisposing factors.

\section{Consent}

Written informed consent was obtained from the patient for publication of this case report and any accompanying images. A copy of the written consent is available for review by the Editor-in-Chief of this journal.

\footnotetext{
Author details

'Department of Microbiology, Queen's Medical Centre, Derby road, Nottingham, NG7 2UH, UK. ${ }^{2}$ Department of Radiology, Queen's Medical
}

Centre, Derby road, Nottingham, NG7 2UH, UK. ${ }^{3}$ Department of Neurosurgery, Queen's Medical Centre, Derby road, Nottingham, NG7 2UH, UK.

\section{Authors' contributions}

WW and TT participated in the concept and design of the manuscript, acquisition of data, and drafting of the manuscript. All authors read and approved the final manuscript.

\section{Competing interests}

The authors declare that they have no competing interests.

Received: 21 January 2011 Accepted: 12 May 2011

Published: 12 May 2011

\section{References}

1. Collins MD, Jones D, Schofield GM: Reclassification of 'Corynebacterium haemolyticum' (MacLean, Liebow \& Rosenberg) in the genus Arcanobacterium gen.nov. as Arcanobacterium haemolyticum nom.rev., comb.nov. J Gen Microbiol 1982, 128(6):1279-1281.

2. Waagner DC: Arcanobacterium haemolyticum: biology of the organism and diseases in man. Pediatr Infect Dis J 1991, 10(12):933-939.

3. Ceilley Rl: Foot ulceration and vertebral osteomyelitis with Corynebacterium haemolyticum. Arch Dermatol 1977, 113(5):646-647.

4. Vargas J, Hernandez M, Silvestri C, Jimenez O, Guevara N, Carballo M, Rojas N, Riera J, Alayo E, Fernandez M, et al: Brain abscess due to Arcanobacterium haemolyticum after dental extraction. Clin Infect Dis 2006, 42(12):1810-1811.

5. Alos Jl, Barros C, Gomez-Garces JL: Endocarditis caused by Arcanobacterium haemolyticum. Eur J Clin Microbiol Infect Dis 1995, 14(12):1085-1088.

6. Chandrasekar PH, Molinari JA: Corynebacterium hemolyticum bacteremia with fatal neurologic complication in an intravenous drug addict. Am J Med 1987, 82(3 Spec No):638-640.

7. Worthington MG, Daly BD, Smith FE: Corynebacterium hemolyticum endocarditis on a native valve. South Med J 1985, 78(10):1261-1262.

8. Meyer DK, Reboli AC: Other Coryneform Bacteria and Rhodococcus. In Principles and Practice of Infectious Diseases. Volume 2.. sixth edition. Edited by: Mandell GL, Bennett JE, Dolin R. Philadelphia: Elsevier Churchill Livingstone; 2005:2465-2478.

9. Maclean PD, Liebow AA, Rosenberg AA: A hemolytic corynebacterium resembling Corynebacterium ovis and Corynebacterium pyogenes in man. J Infect Dis 1946, 79:69-90.

10. Mackenzie A, Fuite LA, Chan FT, King J, Allen U, MacDonald N, DiazMitoma F: Incidence and pathogenicity of Arcanobacterium haemolyticum during a 2-year study in Ottawa. Clin Infect Dis 1995, 21(1):177-181

11. Skov RL, Sanden AK, Danchell VH, Robertsen K, Ejlertsen T: Systemic and deep-seated infections caused by Arcanobacterium haemolyticum. Eur J Clin Microbiol Infect Dis 1998, 17(8):578-582.

12. Parija SC, Kaliaperumal V, Kumar SV, Sujatha S, Babu V, Balu V: Arcanobacterium haemolyticum associated with pyothorax: case report. BMC Infect Dis 2005, 5:68.

13. Cummings LA, Wu WK, Larson AM, Gavin SE, Fine JS, Coyle MB: Effects of media, atmosphere, and incubation time on colonial morphology of Arcanobacterium haemolyticum. J Clin Microbiol 1993, 31(12):3223-3226.

14. Therriault $B L$, Daniels $L M$, Carter $Y L$, Raasch RH: Severe sepsis caused by Arcanobacterium haemolyticum: a case report and review of the literature. Ann Pharmacother 2008, 42(11):1697-1702.

15. Carlson P, Lounatmaa K, Kontiainen S: Biotypes of Arcanobacterium haemolyticum. J Clin Microbiol 1994, 32(7):1654-1657.

16. Carlson P, Kontiainen S, Renkonen OV: Antimicrobial susceptibility of Arcanobacterium haemolyticum. Antimicrob Agents Chemother 1994, 38(1):142-143.

17. Nyman M, Banck G, Thore M: Penicillin tolerance in Arcanobacterium haemolyticum. J Infect Dis 1990, 161(2):261-265.

doi:10.1186/1476-0711-10-17

Cite this article as: Wong et al:: Infective endocarditis caused by

Arcanobacterium haemolyticum: a case report. Annals of Clinical

Microbiology and Antimicrobials 2011 10:17. 